\title{
Interneuronal Activity in Reflex Pathways from Group II Muscle Afferents Is Monitored by Dorsal Spinocerebellar Tract Neurons in the Cat
}

\author{
Elzbieta Jankowska and Anna Puczynska \\ Department of Physiology, Sahlgrenska Academy, Göteborg University, 40530 Göteborg, Sweden
}

\begin{abstract}
The main aim of the study was to investigate whether group II muscle afferents contribute to the inhibition of dorsal spinocerebellar tract (DSCT) neurons and thereby modulate information provided by these neurons in the cat. In intracellular recordings, we found disynaptic IPSPs from group II afferents in the majority of DSCT neurons, most often in parallel with IPSPs evoked from group I afferents. In an attempt to identify interneurons that mediate these IPSPs, the second aim of the study, laminas IV-VII in midlumbar segments were searched for interneurons antidromically activated by stimuli applied within Clarke's column. Such interneurons were found in regions in which focal field potentials were evoked by group I and II afferents, or ventral to them, and most were coexcited by these afferents. The input to these interneurons and their location indicate that they belonged to the previously identified population of premotor interneurons in disynaptic pathways between group I and II afferents and hindlimb motoneurons. The study leads thus to the conclusion that inhibitory actions of group II afferents on DSCT neurons are collateral to actions on motoneurons and that DSCT neurons monitor inhibitory actions of group II afferents on motoneurons as closely as they monitor actions of group I afferents. The results also indicate that interneurons mediating disynaptic reflex actions from tendon organ (group Ib) afferents and those mediating disynaptic actions from secondary muscle spindle (group II) afferents to motoneurons may be parts and parcel of the same interneuronal population rather than constitute distinct interneuronal populations.
\end{abstract}

Key words: spinal cord; interneurons; spinocerebellar neurons; muscle afferents; motor system; cat

\section{Introduction}

Cells of origin of the dorsal spinocerebellar tract (DSCT neurons) have been long recognized as one of the main types of neurons forwarding proprioceptive information to the cerebellum and other parts of the brain. As established previously, information from proprioceptors reaches DSCT neurons both directly and indirectly, i.e., via monosynaptic actions of primary afferents and via spinal interneurons (for review, see Bosco and Poppele, 2001). However, most studies focused on direct input to DSCT neurons, and very little is still known about interneurons that provide indirect input to them. A contribution of interneurons mediating Ia reciprocal inhibition between flexors and extensors has been excluded by both Lindström and Takata (1977) and Hongo et al. (1983b), but interneurons in pathways from both group Ia and Ib afferents have been shown to mediate inhibition of DSCT neurons as well as of motoneurons (Hongo et al., 1983a,b). Disynaptic inhibition of DSCT neurons has also been proposed to be evoked by interneurons in reflex pathways from

Received Nov. 19, 2007; revised Feb. 29, 2008; accepted March 3, 2008.

This work was supported by National Institutes of Health-National Institute of Neurological Disorders and Stroke Grant R01 NS040863. We thank Rauni Larsson for her invaluable assistance and Drs. S. A. Edgley and T. Hongo for their comments on this manuscript.

Correspondence should be addressed to Elzbieta Jankowska, Department of Physiology, Sahlgrenska Academy, Medicinaregatan 11, Box 432, 40530 Göteborg, Sweden. E mail: elzbieta.jankowska@physiol.gu.se.

DOI:10.1523/JNEUROSCI.0466-08.2008

Copyright $\odot 2008$ Society for Neuroscience $\quad$ 0270-6474/08/283615-08\$15.00/0 group II muscle afferents (Bloedel and Courville, 1981, their Fig. 5 ), but this proposal has remained hypothetical ever since it was put forward (Bosco and Poppele, 2001), and early observations by Jansen et al. (1967) suggested that inhibition of DSCT neurons by muscle stretches is mediated by group Ia rather than by group II muscle spindle afferents. Using intracellular records from DSCT neurons (Curtis et al., 1958; Eccles et al., 1961) demonstrated inhibition from group I afferents but did not investigate effects from group II afferents, and only scanty observations link inhibition of DSCT neurons with group II afferents, whether activated electrically (Hongo et al., 1983b, their Fig. 2) or by passive or active muscle stretches (Bosco and Poppele, 2001).

The aim of the present study has therefore been twofold: (1) to find out whether group II afferents provide disynaptic inhibitory input to DSCT neurons, and, if so, (2) to investigate whether inhibitory actions from group II afferents might be evoked by interneurons coexcited by group I and II afferents (represented by interneurons labeled B in Fig. 1) or by interneurons in a more "private" pathway (represented by interneurons labeled A in Fig. 1). To answer these questions, two experimental approaches were used. One involved intracellular records from DSCT neurons in Clarke's column and analysis of the origin of IPSPs evoked in them by electrical stimulation of peripheral nerves. The second approach involved searching for interneurons antidromically activated by stimuli applied within Clarke's column and investigating whether any activated by group II afferents were coexcited by 
group I afferents. The survey included interneurons located within the dorsal horn as well as within the intermediate zone and in the ventral horn (Cavallari et al., 1987; Edgley and Jankowska, 1987b; Bannatyne et al., 2003, 2006).

\section{Materials and Methods}

Preparation. The experiments were performed on four deeply anesthetized cats weighing $2.4-3.2 \mathrm{~kg}$. Anesthesia was induced with sodium pentobarbital (40-44 mg/kg, i.p.; Apoteksbolaget, Göteborg, Sweden) and maintained with intermittent doses of $\alpha$-chloralose $(5 \mathrm{mg} / \mathrm{kg}$; administered i.v., every $1-2 \mathrm{~h}$ up to $50 \mathrm{mg} / \mathrm{kg}$; sanofi-aventis, Bridgewater, $\mathrm{NJ})$. Additional doses of $\alpha$-chloralose were given when increases in continuously monitored blood pressure or heart rate were evoked by peripheral or central stimulation or if the pupils dilated. During recordings, neuromuscular transmission was blocked by pancuronium bromide $(\mathrm{Pa}-$ vulon, $\sim 0.2 \mathrm{mg} \cdot \mathrm{kg}^{-1} \mathrm{~h}^{-1}$, i.v.; Organon, Göteborg, Sweden), and the animals were artificially ventilated. The effectiveness of synaptic transmission was increased by intravenous application of 4-AP in doses $0.1-$ $0.2 \mathrm{mg} / \mathrm{kg}$ (intravenously). Mean blood pressure was kept at 100-130 $\mathrm{mmHg}$ and the end-tidal concentration of $\mathrm{CO}_{2}$ at $\sim 4 \%$ by adjusting the parameters of artificial ventilation and the rate of a continuous infusion of a bicarbonate buffer solution with $5 \%$ glucose $\left(1-2 \mathrm{mg} \cdot \mathrm{kg}^{-1} \mathrm{~h}^{-1}\right)$. Core body temperature was kept at $\sim 38^{\circ} \mathrm{C}$ by servo-controlled infrared lamps. The experiments were terminated by a lethal dose of anesthetic. All these procedures were approved by the local ethics committee (Göteborgs djurförsöksetiska nämnd, Göteborg, Sweden) and followed National Institutes of Health and European Union guidelines for animal care.

The spinal cord was exposed by laminectomy from the second to the sixth lumbar (L2-L6) segments and at the level of the low thoracic (Th10-Th12) segments. Records from the DSCT cells were obtained from Clarke's column in the L3 and L4 segments. Interneurons activated from Clarke's column were searched for in the L4 through L6 segments. In both cases, the dura mater over the lumbosacral enlargement was left intact except for small holes $\left(\sim 1 \mathrm{~mm}^{2}\right)$ through which the microelectrodes were advanced. A somewhat larger hole was made over the caudal part of the L4 segment to allow transection of the dorsal columns. The transection of the dorsal columns at this level was made to eliminate, or at least reduce, direct synaptic input to DSCT neurons and thus ease detection of any IPSPs that might otherwise be superimposed on monosynaptic EPSPs from peripheral afferents following the procedure of Hongo et al. (1983a,b). However, because the access to the dorsal columns in the L4 was limited by the trajectory of the blood vessels, only partial lesions were made so that the spinal circulation would be only minimally interfered with. This abolished monosynaptic EPSPs evoked by afferent fibers in the majority but not all of the DSCT neurons. The dorsal columns were also transected rostral to the recording site (at Th12 level) to prevent synaptic activation of DSCT neurons by spread of current to afferent fibers in the dorsal columns when stimuli were applied to the ipsilateral lateral funiculus at Th10 level to activate them antidromically.

The following left hindlimb nerves were dissected free, transected, and mounted on stimulating electrodes: quadriceps (Q) and sartorius (Sart) branches of the femoral nerve mounted in subcutaneous cuff electrodes, the posterior biceps and semitendinosus (PBST), anterior biceps and semimembranosus (ABSM), gastrocnemius-soleus (GS), plantaris (PL), flexor digitorum and hallucis longus (FDL), and deep peroneal (DP), including extensor digitorum longus and tibialis anterior nerves.

Stimulation and recording. Peripheral nerves were stimulated with constant voltage stimuli at intensities expressed in multiples of threshold (T) for the activation of the most excitable fibers. Axons of DSCT neurons within the lateral funiculus at Th10 level were stimulated extradurally, with two silver ball electrodes in contact with its surface, using 0.2 -ms-long constant current stimuli of 100-300 $\mu \mathrm{A}$. Axons of interneurons projecting to Clarke's column were stimulated through tungsten electrodes placed at locations at which distinct antidromic field potentials were evoked by Th stimuli and at which DSCT neurons responded to stimulation of group I afferents of the Q and/or GS nerves. This was established using a glass micropipette and confirmed after insertion of the tungsten electrode by recording through it. Glass micropipettes filled with $2 \mathrm{M}$ solution of $\mathrm{NaCl}(1.5-3 \mathrm{M} \Omega)$ or with $2 \mathrm{M}$ solution of potassium citrate $(2-5 \mathrm{M} \Omega$ ) were used for extracellular recording from the interneurons and for intracellular recording from DSCT neurons, respectively. DSCT neurons were searched for within the boundaries of Clarke's column that were originally defined in the same way. The neurons recorded from before transection of the dorsal columns caudal to the Clarke's column were identified by both their monosynaptic input from group Ia afferents and antidromic activation from the lateral funiculus at Th10 level, stimulated as described above, but those recorded after transection only by the latter criterion.

Analysis. Both original data and averages of 10-30 single records (with the time resolution of $30 \mu$ s per address) were stored on-line using software for sampling and analysis developed by E. Eide, T. Holmström, and N. Pihlgren (Göteborg University, Göteborg, Sweden). The latencies of postsynaptic potentials were measured from afferent volleys recorded from the cord dorsum close to the recording electrode penetration site and of antidromic spikes from the stimulus artifacts. Differences between datasets were assessed for statistical significance by using Student's $t$ test (for unpaired samples assuming equal variances and two-tailed distribution) and Mann-Whitney nonparametric test.

\section{Results}

\section{IPSPs evoked in DSCT neurons}

Stimulation of group II afferents in hindlimb nerves evoked IPSPs in the majority of the 41 investigated DSCT neurons. The sample included DSCT neurons in which oligosynaptic IPSPs were induced from at least one of the muscle nerves and in which no action potentials interfering with the analysis were generated during testing. The analysis started when the characteristic spontaneous firing of the penetrated DSCT neurons stopped by itself or after the neurons were hyperpolarized (by 10-20 nA current passed through the recording electrode) or were depolarized (by 5-25 nA) until the voltage-sensitive $\mathrm{Na}$ channels were inactivated. Depolarization was preferred because it increased the size of the IPSPs. When recording conditions became stable, a survey of the effects of stimulation of all of the nerves at five times threshold was first made and was followed by graded stimulation of the nerves from which the IPSPs were evoked to find out whether these were induced by group I or II afferents (Fig. 1).

Any IPSPs evoked at stimulus intensities subthreshold for group II afferents (up to 1.7-2T, depending on the nerve) (Jack, 1978) were classified as having been evoked from group Ib (or both Ia and Ib) afferents, with examples shown in Figure 2, $A$ and $C$. These IPSPs were evoked at latencies (mean, $2.17 \pm 0.05 \mathrm{~ms}$ ) (Fig. $3 A$ ) that fell within the range (1.54-2.62 ms) reported previously for IPSPs of group I origin (Hongo et al., 1983a). Only such IPSPs were found in 16 of $82(19.5 \%)$ tests in which IPSPs were induced by stimulation of any of the muscle nerves.

We attributed IPSPs to group II afferents if they appeared when the stimulus intensity was raised above the intensity supramaximal for group I afferents (as judged from the amplitudes of afferent volleys) and was well above the threshold for group II afferents. Another requirement was that the IPSPs should grow when the stimulus intensity increased between $2 \mathrm{~T}$ and $5 \mathrm{~T}$. The latencies of these IPSPs were generally longer than latencies of IPSPs evoked by group I afferents, on average $3.54 \pm 0.09 \mathrm{~ms}$, within the range of $2.43-5.48 \mathrm{~ms}$ with respect to group I afferent volleys. In individual neurons, latencies of IPSPs of group II origin exceeded those of group I origin by $0.97-2.11 \mathrm{~ms}$ (Fig. $3 C$, light bars).

IPSPs attributable to group II afferents were the only effects of stimulation of a muscle nerve in 28 of $82(34.1 \%)$ tests, as in the DSCT neuron illustrated in Figure $2 F-H$. IPSPs from group II 


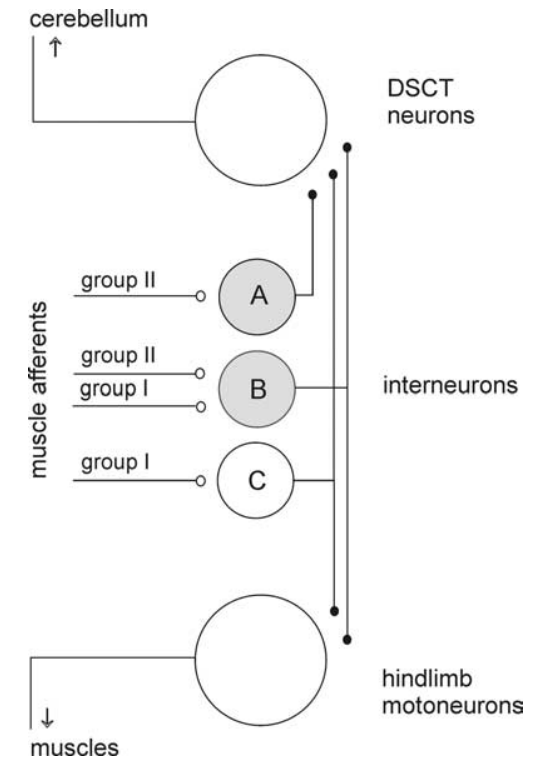

Figure 1. Diagram of hypothetical coupling between group I and II muscle afferents and DSCT neurons investigated in this study. " $A$ " and " $B$ " denote interneurons that might mediate inhibitory actions of group II afferents on DSCT neurons. Of these, " $A$ " represents interneurons in private pathways between group II afferents and DSCT neurons, and " $B$ " represents interneurons in shared pathways from group I and II afferents and acting on both DSCT neurons and hindlimb $\alpha$ motoneurons. " $C$ " represents interneurons in pathways from group I afferents that were shown previously to inhibit both DSCT neurons and hindlimb $\alpha$ motoneurons (Hongo et al., 1983a,b). The findings of this study provide evidence for the most common coupling between group II afferents and DSCT neurons via interneurons "B."
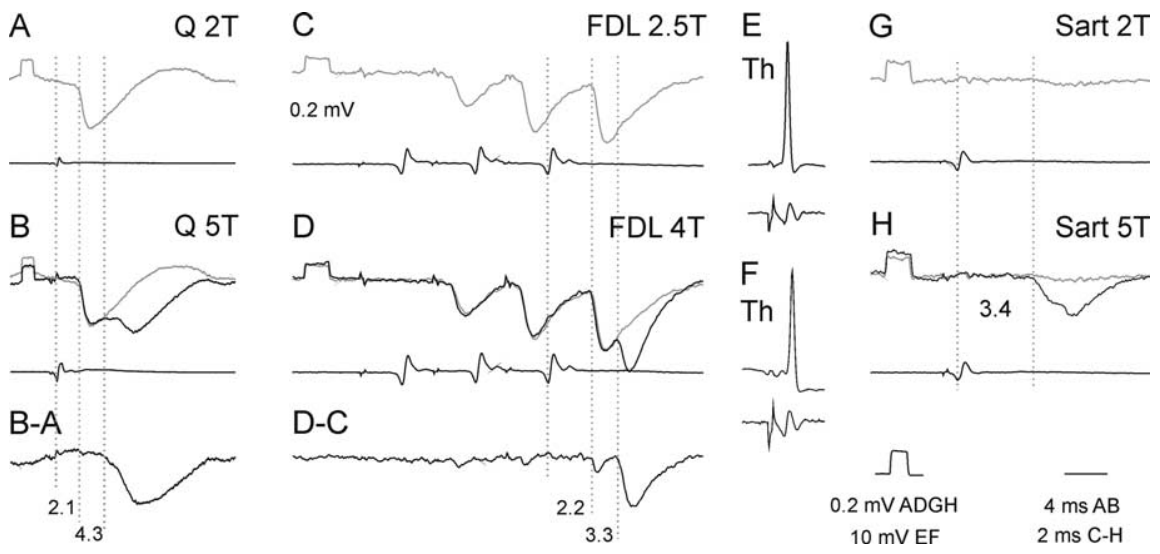

Figure 2. Comparison of IPSPs evoked by stimuli subthreshold and near-maximal for group II afferents. Top records in each panel are intracellular records from three DSCT neurons, in $\boldsymbol{A}, \boldsymbol{B}, \boldsymbol{C}-\boldsymbol{E}$, and $\boldsymbol{F}-\boldsymbol{H}$, respectively. Bottom records are potentials from the surface of the spinal cord. IPSPs evoked by stimuli activating mainly group I afferents (gray) and both group I and group II afferents (black) are shown separately and superimposed on each other. Records below $\boldsymbol{B}$ and $\boldsymbol{D}$ show differences between them. Dotted lines indicate onsets of the afferent volleys and onsets of the IPSPS attributable to group I and group II afferents. The numbers to the left of the dotted lines indicate their latencies in milliseconds. $\boldsymbol{E}$ and $\boldsymbol{F}$ show antidromic spikes evoked after penetration of the neurons. Rectangular calibration pulses at the beginning of intracellular records, $0.2 \mathrm{mV}$. Voltage calibration and time calibration below $\boldsymbol{H}$ are as indicated. In this and in the following figures, the negativity in intracellular records is downward and in records from the cord dorsum is upward.

afferents that followed IPSPs evoked by group I afferents were found in 20 of $82(24.4 \%)$ tests. They were evoked by single stronger stimuli, as in the neuron illustrated in Figure 2, $A$ and $B$, or only after the second or third stimulus in the train, as in Figure $2 D$. They are represented by IPSPs appearing at the level of rightmost dotted lines in these figures.

IPSPs evoked in several DSCT neurons were more difficult to attribute unequivocally to group I or II afferents because they started to appear at stimulus intensities that were unlikely to be sufficient to activate group II afferents (e.g., 1.5T for Q), which would indicate that these IPSPs were evoked by group I afferents, but their latencies exceeded those of IPSPs of undisputable group origin, and their amplitudes increased with stimulus intensity with similar latencies, as illustrated in Figure 4. In view of their ow thresholds, the early components of these IPSPs were considdo have been evoked by group I afferents, but at longer than stimulus intensities as IPSPs of group II origin. Such IPSPs contituted $21.9 \%$ ( 18 of 82 ) of the total number of IPSPs.

With the latter IPSPs classified as being of both group I and II Wrin, IPSPs from group II afferents of any nerves were evoked in $92.7 \%$ (38 of 41) of DSCT neurons of our sample and IPSPs from group I afferents in $75.6 \%$ (31 of 41 ) neurons.

The peak amplitudes of IPSPs attributable to group II afferents were usually comparable with, or smaller than, the amplides of IPSPs evoked by group I afferents. As shown in Figure 3, $B$ and $D$, both were on average $\sim 0.5 \mathrm{mV}$, but their values are underestimated because the state of the neurons often deterioturing recording.

The origin of IPSPs evoked from group I afferents in individSCT neurons was as diversified as in the sample of Hongo et from several nerves, most frequently from $\mathrm{Q}$ and GS or from $\mathrm{Q}$ and FDL. The origin of IPSPs evoked from group II afferents likewise varied, but they were most frequently evoked from other combinations of afferents: Q, Sart, and DP; Q, FDL, and DP; Q and Sart; or Q and DP.

\section{Interneurons projecting to Clarke's column}

While searching for interneurons antidromically activated from Clarke's column, stimuli were applied via a tungsten electrode inserted as described in Materials and Methods. As a search stimulus, we used $50 \mu \mathrm{A}$ (with the estimated current spread within $0.2-0.3 \mathrm{~mm}$ radius from the electrode tip) (Gustafsson and Jankowska, 1976). Assuming that IPSPs from group I and/or II afferents are evoked in DSCT neurons located within a length of $\sim 10$ mm of Clarke's column, that these IPSPs are evoked by a few interneurons (Hongo et al., 1983a), and that at least some interneurons form synaptic contacts at more than one location, we estimated that at least $10-20 \%$ of interneurons projecting to Clarke's column would be activated by such stationary electrodes and that we might find $7-15$ of $\sim 75$ interneurons in the L5 segment estimated to project to Clarke's column (Hongo et al., 1983a) and a similar number in the L4 segment. The search for interneurons was made in several electrode tracks 50-100 $\mu \mathrm{m}$ apart. The rationale for choosing such intertrack distances was that spike potentials of individual interneurons may be detected at up to $100 \mu \mathrm{m}$ distances, provided that low-impedance electrodes are used and that recording from neurons detected at the longest distances may be improved by subsequently replacing the 

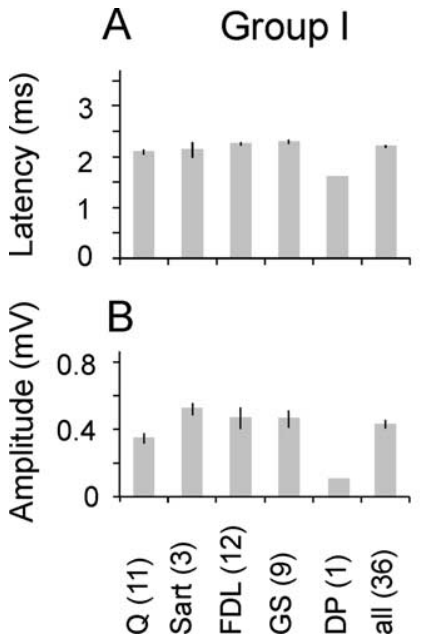
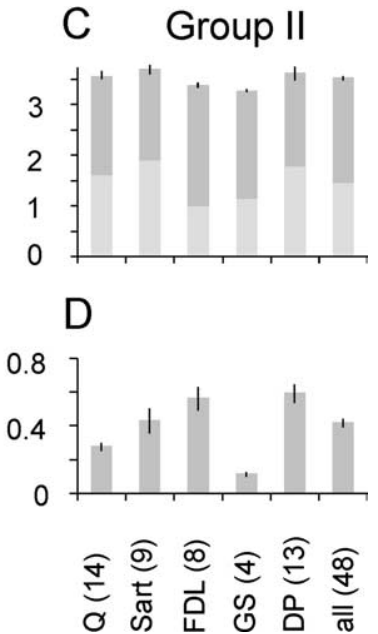

Figure 3. Comparison of latencies and peak amplitudes of IPSPs attributable to either group I or II afferents of the quadriceps, sartorius, flexor digitorum and hallucis longus, gastrocnemius-soleus, and deep peroneal nerves and pooled data for IPSPs evoked from any of these nerves. The data are derived from the DSCT neurons in which the amplitudes of IPSPS evoked by group I $(\boldsymbol{A}, \boldsymbol{B})$ and/or group II $(\boldsymbol{C}, \boldsymbol{D})$ afferents were of at least $0.1 \mathrm{mV}$ and could be separated using subtraction procedures illustrated in Figure 2. The data for IPSPs recorded in additional 17 tests in which neither the latency nor amplitude of IPSPs of group I or group II origin could be measured, as of those illustrated in Figure 4, have been excluded. Dark shaded bars, Means and SEM for IPSPs evoked from the nerves indicated below $\boldsymbol{B}$ and $\boldsymbol{D}$, with the number of IPSPs in parentheses. Lighter parts of bars in $\boldsymbol{C}$ show mean differences between latencies of IPSPs evoked from group I and II afferents in neurons in which both were evoked. No statistically significant differences were found between amplitudes of IPSPs evoked by group I and II afferents of the same nerve. No statistically significant differences were either found between effects evoked by stimulation of the same group of afferents of different nerves. Student's $t$ test and Mann-Whitney tests gave similar results.

electrodes. The explored areas covered the width of the gray matter within which focal field potentials were evoked from group I and/or II afferents.

Using this approach in two experiments, we found 43 extracellularly recorded interneurons that were antidromically activated from Clarke's column. Thirty-one of these neurons showed synaptic input from the stimulated muscle nerves; 20 interneurons responded to stimulation of group I as well as group II afferents, seven interneurons were activated only by stimuli near maximal for group II afferents, and four interneurons were activated only by group I afferents. Input from group II afferents was most frequently from Q $(n=23)$ and less frequently from Sart $(n=12)$, DP $(n=7)$, and FDL $(n=5)$. Input from group I afferents was likewise most frequently from Q $(n=14)$ and less frequently from PL $(n=6)$, FDL $(n=5)$, Sart $(n=5)$, GS $(n=$ $2), \operatorname{DP}(n=2), \operatorname{PBST}(n=1)$, and $\operatorname{ABSM}(n=1)$.

All these neurons were found within the intermediate zone, at locations at which extracellular field potentials were evoked from both group I and II afferents or only group II afferents (at depths 2.15-2.9 mm from the cord dorsum). Conversely, we have not encountered any midlumbar interneurons antidromically activated from Clarke's column within the dorsal horn.

The records in Figure $5 E$ illustrate antidromic activation of one of the interneurons that was coexcited by group I and II afferents (at a latency of $2.1 \mathrm{~ms}$ ) and the collision of antidromic spikes after synaptic activation of this neuron at sufficiently short intervals (twice antidromic conduction time plus refractory period of the axons) (Fuller and Schlag, 1976; Asif and Edgley, 1992; Krutki et al., 2003). The series of records in $A-D$ show responses that were evoked by $\mathrm{Q}$ stimulation at increasing intensities, from
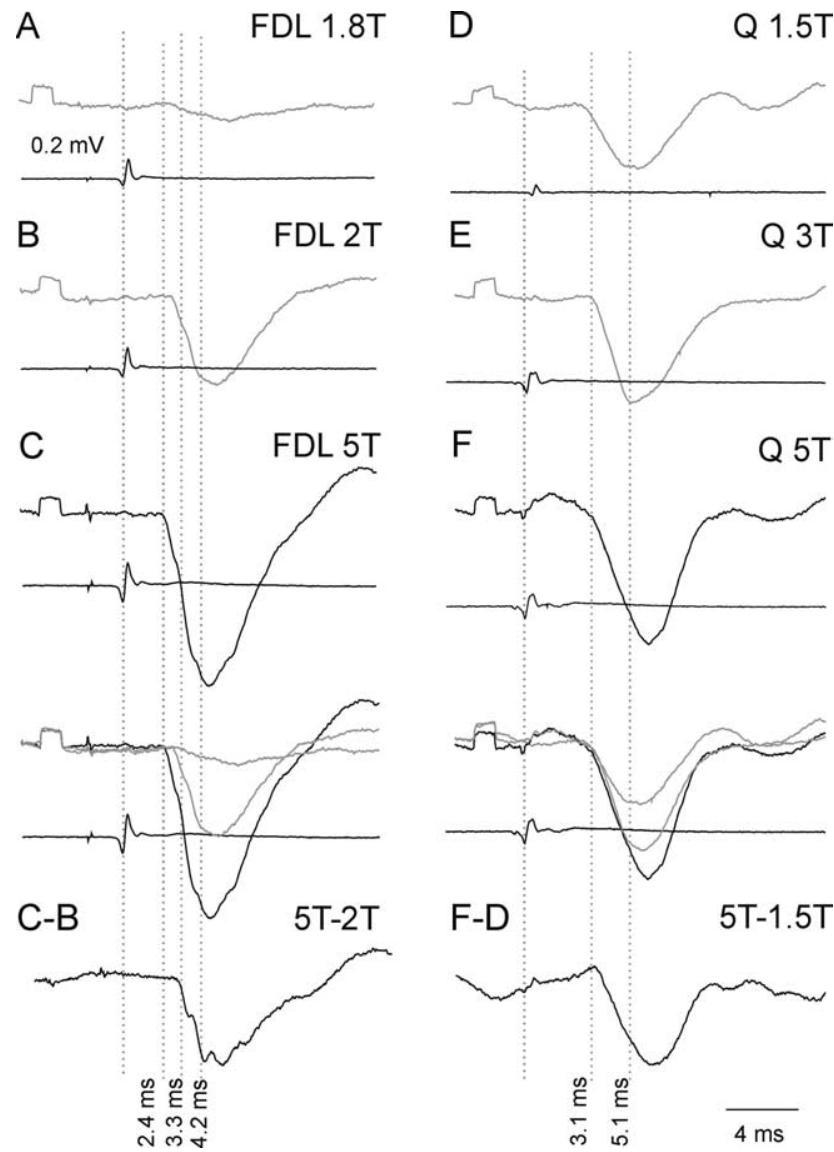

Figure 4. Examples of IPSPS most likely evoked by both group I and II afferents, at similar latencies but with components attributable to group II origin added with increasing stimulus intensity. Records from two DSCT neurons, in $\boldsymbol{A}-\boldsymbol{C}$ and $\boldsymbol{D}-\boldsymbol{F}$, respectively, and from the surface of the spinal cord. Similar format as in Figure 2. Note that the IPSPs evoked by 5 T stimuli in $C$ and Fare shown twice, either separately or together with IPSPs evoked by weaker stimuli. Note also that they grew almost continuously with stimulus intensity and that later components evoked by stronger stimuli might be distinguished in $\boldsymbol{C}$ but not in $\boldsymbol{F}$.

submaximal for group I afferents $(A, B)$, near maximal for group I afferents and exceeding threshold for group II afferents $(C)$, and near maximal for group II afferents $(D)$. The neuron did not respond to single stimuli, and responses to the second of a pair of stimuli appeared at latencies of 1.3-2.3 ms from group I volleys. Single spikes were evoked during the first (group I) component of the focal field potentials by weaker stimuli $(A-C)$ and a burst of spikes at 2.8-5 ms latencies during the second (group II) component of these potentials by stronger stimuli $(D)$. The ranges of latencies of the earliest spikes evoked by group I and II afferents are indicated above records in $B-D$ by short and long horizontal lines, respectively. Minimal segmental latencies of activation of other interneurons were $0.6-1.7 \mathrm{~ms}$ from group I afferents and $1.8-3.8 \mathrm{~ms}$ from group II afferents.

Responses of interneurons that were found to be activated by group II but not by group I afferents are illustrated in Figure 6 . Also in this case, the interneuron responded only after the second stimulus, but, in contrast to that illustrated in Figure 5, its activation required stimuli exceeding $3 \mathrm{~T}$, and it responded at longer latencies; spikes evoked at these latencies coincided with field potentials evoked by group II afferents. Such neurons were classified as belonging to interneurons in more private pathways from group II afferents, represented by cell A in Figure 1. However, it could not be excluded that they were coactivated by group 


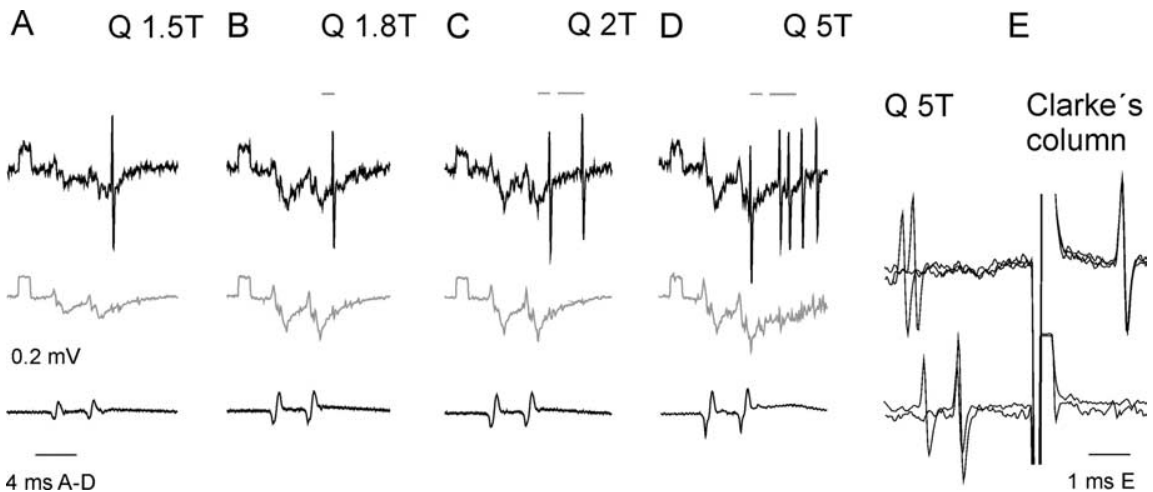

Figure 5. Examples of extracellular records from an interneuron coexcited by group I and II afferents. Top traces in $\boldsymbol{A}-\boldsymbol{D}$ and all those in $\boldsymbol{E}$ are single extracellular records from an interneuron located within the intermediate zone of the L 4 segment (depth 2.3 $\mathrm{mm}$ from the surface of the spinal cord). Middle traces in $\boldsymbol{A}-\boldsymbol{D}$ are 10 averaged records of field potentials, whereas bottom traces are cord dorsum potentials. $\boldsymbol{A}-\boldsymbol{D}$, Responses evoked by increasing intensities of stimulation of the $Q$ nerve. Horizontal lines above the records in $\boldsymbol{B}-\boldsymbol{D}$ indicate ranges of minimal latencies of spike potentials evoked by $1.8,2$, and 5 T stimuli. $\boldsymbol{E}$, Superimposed records in which both synaptically and antidromically evoked responses were evoked (to the left and to the right of the stimulus artifacts in the middle of the top traces, respectively) and in which the antidromic responses were collided by synaptically evoked responses induced at sufficiently short intervals.

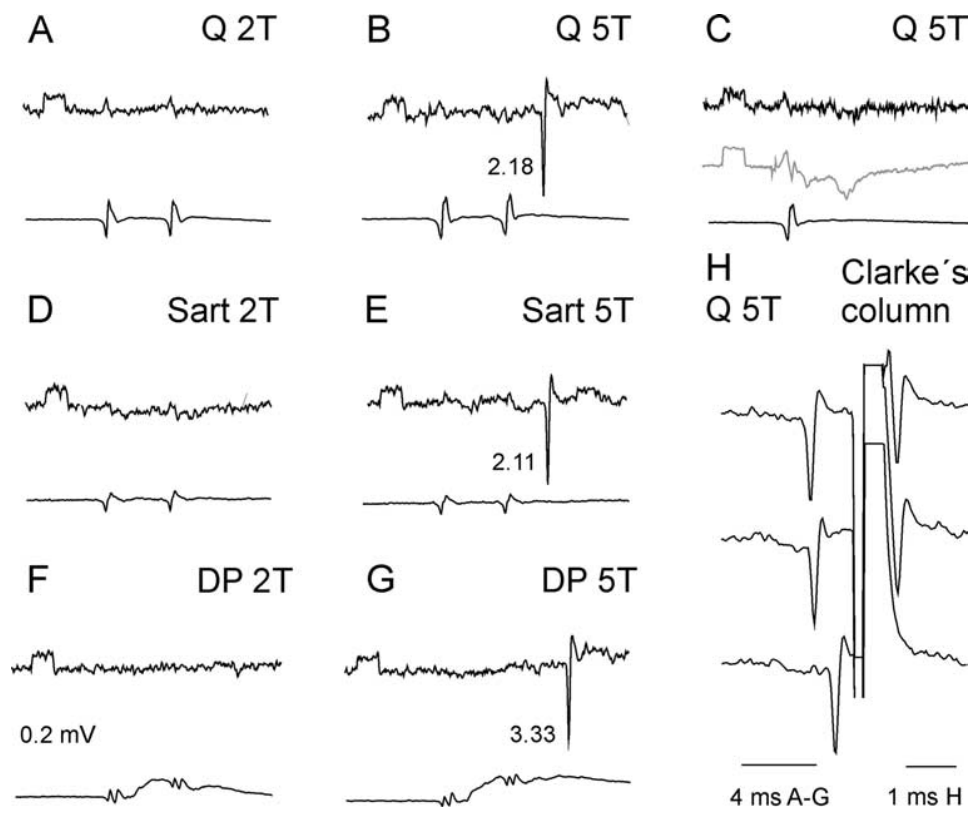

Figure 6. Examples of extracellular records from an interneuron activated by group II afferents. Extracellular records from an interneuron located within the intermediate zone of the $L 4$ segment (depth of $2.3 \mathrm{~mm}$ from the surface of the spinal cord) at a location at which field potentials from both group I and II afferents were very small. $\boldsymbol{A}-\boldsymbol{C}, \boldsymbol{D}, \boldsymbol{E}$, and $\boldsymbol{F}, \boldsymbol{G}$, Responses evoked by stimulation of the Q, Sart, and DP nerves, respectively. Note that the neuron was discharged by two stimuli at $5 T$ but not at $2 \mathrm{~T}$ nor by single stimuli at 5T. The numbers between the records in $\boldsymbol{B}, \boldsymbol{E}$, and $\boldsymbol{G}$ are the response latencies (in milliseconds) with respect to the group l afferent volleys of the effective stimulus. The middle trace in C shows average record of a field potential recorded $\sim 100$ $\mu \mathrm{m}$ from the interneuron. $\boldsymbol{H}$, Collision between synaptically and antidromically evoked spike potentials with stimulus artifacts truncated.

I afferents from some of the peripheral nerves that were not dissected in these experiments and belong to cells labeled B.

Because the earliest responses of the interneurons were evoked at latencies of 0.6 and $1.8 \mathrm{~ms}$ from afferent volleys evoked by group I and group II afferents, respectively, they could have contributed not only to the later but also to the earliest components of IPSPs evoked in DSCT neurons from these afferents (at latencies of 1.5-2.9 and 2.4-5.8 ms). However, this would require that the conduction times to Clarke's column were sufficiently short. To verify this, the sums of the minimal latencies of synaptic and antidromic activation of individual interneurons of our sam- ple were related to the latencies of IPSPs evoked in DSCT cells. The plots in Figure 7 show that these sums (open triangles in $A$, $B, E, F)$ were generally longer than would be required because their majority exceeded latencies of IPSPs (diamonds).

Several reasons for this mismatch were therefore considered. One was that the timing of actions of the interneurons on DSCT neurons was considerably overestimated. The conduction time along axons of the antidromically activated interneurons would be overestimated by $0.2-0.3$ $\mathrm{ms}$ because of the utilization time needed for electrical stimuli to generate action potentials (Jankowska and Roberts, 1972; Roberts and Smith, 1973). It would also be overestimated by additional $0.2-0.3 \mathrm{~ms}$ if interneurons were activated from more rostral parts of Clarke's column than the regions in which the most caudally sampled DSCT neurons were located and/or by $0.1-0.3 \mathrm{~ms}$ in the case of interneurons located more caudally than those responsible for the earliest IPSPs in DSCT neurons. These differences would be related to conduction velocity of $\sim 10 \mathrm{~m} / \mathrm{s}$ along terminal axon collaterals of the interneurons close to or within Clarke's column (Jankowska and Roberts, 1972) and 20-50 $\mathrm{m} / \mathrm{s}$ over $\sim 5 \mathrm{~mm}$ length of more proximal parts of their axons (Hongo et al., 1983b, their Fig. 7). The sum data points should, conversely, be weighed against synaptic delays of $\sim 0.2 \mathrm{~ms}$ with which the interneurons would induce IPSPs in DSCT neurons. Because all of these time estimates fall within certain ranges, the exact values of the ensuing errors could not be subtracted from the data points representing sums in Figure 7, $A, B, E$, and $F$. We could subtract only an arbitrary total overestimate of these sums $\{(0.3+0.3+$ $0.3)-0.2\}=0.7 \mathrm{~ms}$ ) by shifting them down in $C, D, G$, and $H$. This improved the match between the sums and the latencies of IPSPs in DSCT neurons; an even better match was obtained when the data for individual interneurons in $C, D$, and $G$ were in addition shifted to the right, under the assumption that interneurons mediating the shortest-latency IPSPs were not included in our sample. The correspondence between them was then as for the sample of interneurons analyzed by Hongo et al. $(1983 a, b)$. The errors in our estimates of timing of actions of interneurons in pathways between group I and II afferents and DSCT neurons should thus be comparable.

Group I-activated interneurons projecting to Clarke's column appeared previously to be primarily, if not exclusively, inhibitory because any interneuronally mediated EPSPs from group I afferents were seen in DSCT neurons only if transection of the dorsal columns caudal to Clarke's column was incomplete (Hongo et al., 1983a). In addition, only IPSPs were evoked in hindlimb mo- 
toneurons by stimuli applied in Clarke's column or in DSCT neurons by stimuli applied in motor nuclei (Hongo et al., 1983b). In the present sample of DSCT neurons, EPSPs from group II afferents were also seen only occasionally (in 4 of 41 neurons). These EPSPs were evoked from the same nerves from which small monosynaptic EPSPs were evoked in the same experiment and may therefore be attributed to interneurons located rostral to the lesion that were activated by undamaged afferents and that might not target motoneurons.

\section{Discussion}

The results of this study provide positive answers to both of our two original questions. With respect to the first question, they demonstrate that IPSPs can be evoked in a high proportion of the DSCT neurons by group II afferents, most often in parallel with IPSPs of group I origin. With respect to the second question, they indicate that a high proportion of interneurons likely to mediate these IPSPs are coexcited by group I and II muscle afferents.

\section{Inhibition of DSCT neurons from group} II muscle afferents

Latencies of IPSPs evoked by group II afferents in the present sample of DSCT neurons were $\sim 1.5 \mathrm{~ms}$ longer than of IPSPs evoked by group I afferents. However, when differences in peripheral conduction time of group I and II afferents (1-1.5 ms) (Edgley and Jankowska, 1987a; Riddell et al., 1993, 1995) are taken into account, the segmental latencies of IPSPs attributable to group II afferents, i.e., latencies from group II volleys, are comparable with the segmental latencies of IPSPs of group I origin.

While estimating coupling of IPSPs evoked in DSCT neurons, one should also note that latencies of these IPSPs were by $\sim 1$ ms longer than latencies of disynaptic actions of group I and II afferents on $\alpha$ motoneurons or on segmental interneurons, reported to be up to $\sim 2 \mathrm{~ms}$ from group I afferents (Eccles et al., 1957) and up to 3-4 ms from group II afferents (Jankowska et al., 2005, their Fig. 2). However, conduction times from the same interneurons to DSCT neurons might exceed those to $\alpha$ motoneurons because of the longer intraspinal distances and/or lower conduction velocity along the ascending than the descending axon collaterals of the interneurons. This is suggested by the longer range of latencies of antidromic activation of our sample of interneurons from Clarke's column (0.9-2.8 ms) (Fig. 7C,D, G,H) than the range of latencies of antidromic activation of another sample of interneu-

B

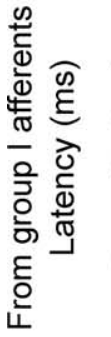

\section{From $Q$ and Sart}

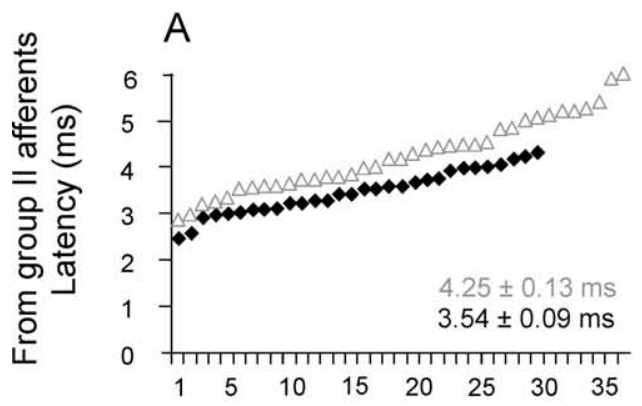

C
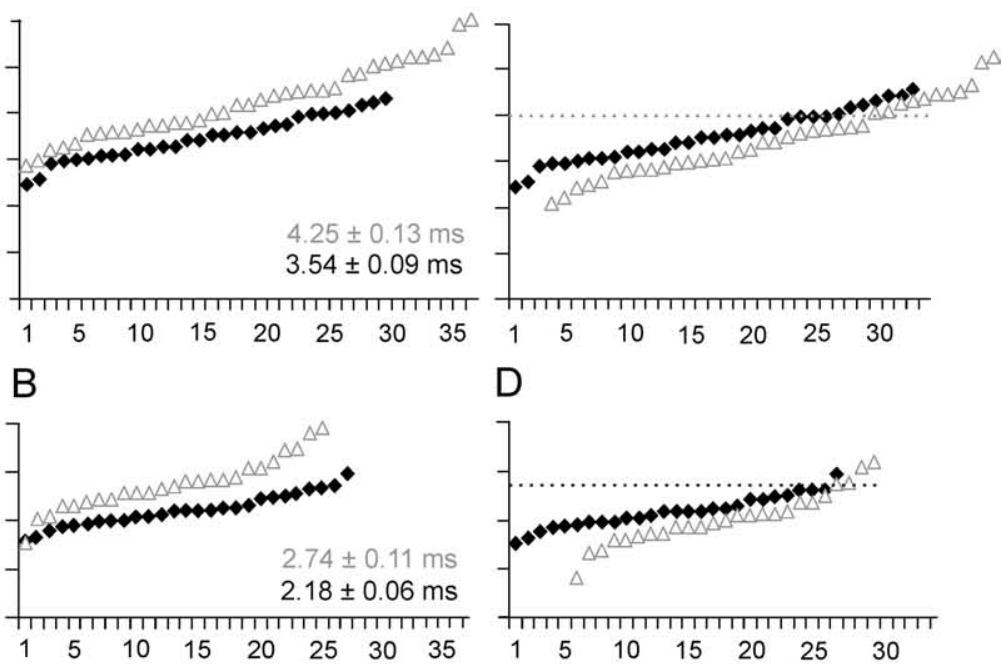

D

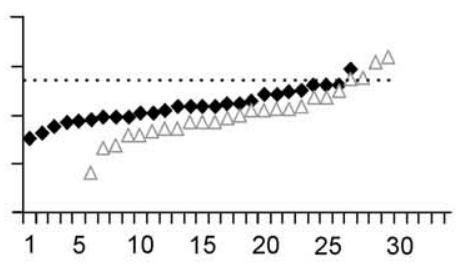

\section{From DP, FDL, GS, PI \& PBST}

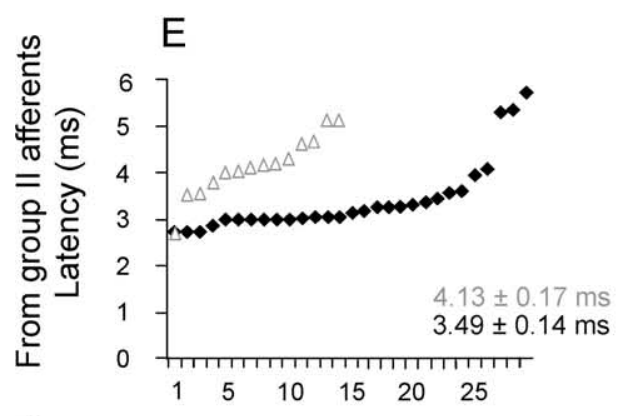

G
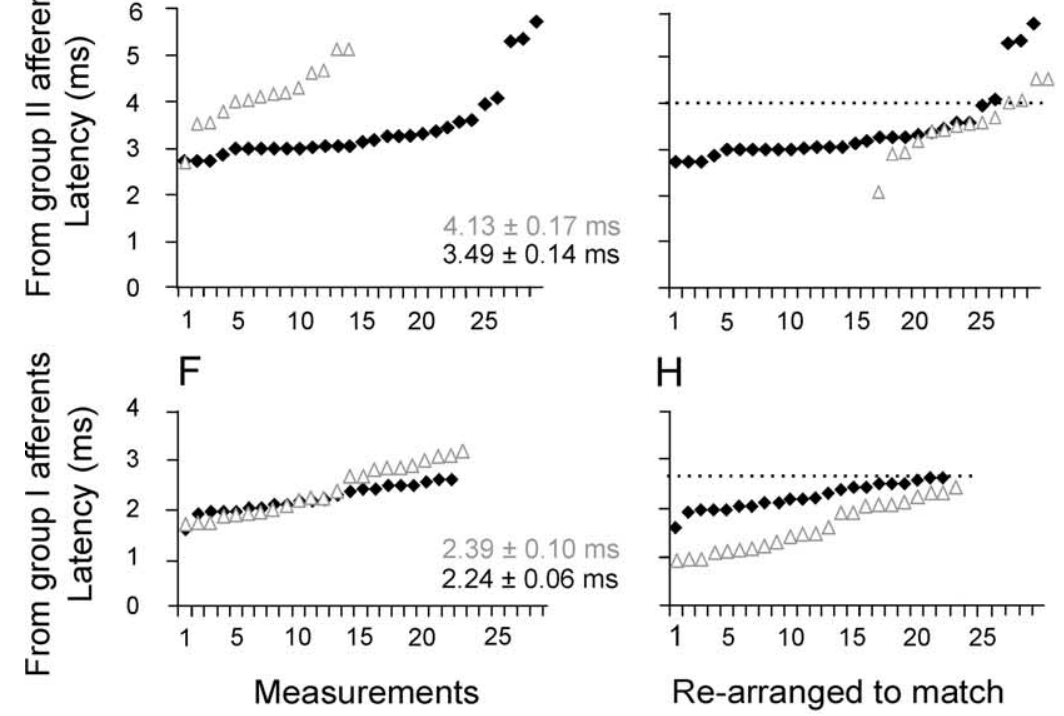

- IPSPs in DSCT neurons

$\triangle$ Sums of latencies of synaptic and antidromic activation of intereurons

Figure 7. Comparison of latencies of IPSPs evoked in DSCT neurons and of activation of interneurons with input from group I and $I I$ afferents. Diamonds in $\boldsymbol{A}-\boldsymbol{D}$ and $\boldsymbol{E}-\boldsymbol{H}$ show latencies of IPSPs evoked in DSCT neurons by stimulation of the more proximal ( $Q$ and Sart) and more distal (DP, FDL, GS, PL, and PBST) nerves, from group II afferents $(\boldsymbol{A}, \boldsymbol{E})$ and from group I afferents $(\boldsymbol{B}, \boldsymbol{F})$. These have been plotted in the ascending order of latency. Dotted lines indicate upper limits of latencies of IPSPs classified with highest confidence as evoked disynaptically. Open triangles in all these plots represent sums of latencies of activation of individual interneurons by group I or II afferents and of antidromic activation of the same interneurons from Clarke's column, plotted according to the ascending order of the sums. In $A, B$ and $E, F$, no corrections have been made to these sums to account for latent period of activation of axons of interneurons by electrical stimuli and other sources of their overestimation (see Results). In $C, D$ and $\mathbf{G}, \boldsymbol{H}$, such corrections have been made, and the data are shifted to the right to match IPSPs mediated by later activated interneurons. For the justification of such rearrangements, see Results. rons at the same segmental location from the hindlimb motor nuclei (0.5-1.6 ms) (Cavallari et al., 1987, their Fig. 4). Latencies of actions of group II afferents on DSCT neurons are thus compatible with disynaptic coupling. By demonstrating them, the 
results of this study thus provide the experimental evidence for the coupling between these afferents and DSCT neurons that was hypothesized by Bloedel and Courville (1981) to be via single inhibitory interneurons. Conversely, our results might be considered as at variance with conclusions of Jansen et al. (1967), who attributed inhibition of discharges of DSCT neurons by muscle stretches to activation of primary (group Ia) rather than secondary (group II) muscle spindle endings. However, the disagreement may in this case be only apparent because only gastrocnemius-soleus muscles were stretched by Jansen et al. (1967), and, according to our data, these muscles are a much weaker source of inhibition of DSCT neurons via group II afferents than the quadriceps, flexor digitorum, and hallucis longus or pretibial flexors.

\section{The same interneurons may mediate inhibition of DSCT neurons by group I and II afferents and may monitor actions of these afferents on hindlimb motoneurons}

The second question addressed by this study was whether inhibition of DSCT neurons by group II afferents is mediated by interneurons that are coexcited by group I and II afferents or by a distinct, "private" population of interneurons with selective input from group II afferents, the two variants indicated in Figure 1. Because the majority (20 of 31 ) of our sample of interneurons with input from peripheral afferents found to project to Clarke's column were activated by both group I and II afferents, the first of these coupling modes appears to be more common. Nevertheless, with some (7 of 31) interneurons activated by group II but not group I afferents, the second coupling mode remains as an alternative possibility. Coactivation by group I and II afferents of the majority of interneurons mediating inhibition of DSCT neurons leads to the further conclusion that IPSPs from group II afferents evoked via these interneurons are collateral to their actions on motoneurons. The conclusion is inferred from two groups of previous findings. The first of these included demonstration that the majority of interneurons coexcited by group I and II afferents in midlumbar segments project to motor nuclei and directly excite or inhibit $\alpha$ motoneurons. This was found both in electrophysiological studies (Cavallari et al., 1987; Edgley and Jankowska, 1987b) and by reconstructing axonal projections of these neurons and by defining transmitter content in their terminals (Bras et al., 1989) (B. A. Bannatyne, T. T. Liu, I. Hammar, K. Stecina, E. Jankowska, and D. J. Maxwell, unpublished observations). The second group of findings on which we base our conclusion provide evidence that the same group I-activated inhibitory intermediate zone interneurons act on both DSCT neurons and hindlimb motoneurons. These findings include the demonstration that monosynaptic IPSPs are evoked in DSCT neurons by stimuli applied in hindlimb motor nuclei and in motoneurons by stimuli applied in Clarke's column, both in parallel with IPSPs evoked by group Ib afferents, and that only group I-activated interneurons inhibiting motoneurons were found to project to the level of Clarke's column (Brink et al., 1983; Hongo et al., 1983a,b). What holds true for actions of interneurons activated by group I afferents should thus also apply to actions of the same interneurons when they are coactivated by group II afferents.

Hongo et al. (1983a,b) reported parallel actions of only group Ib afferents. However, these authors focused on effects evoked by interneurons that were located more caudally (in the L6 and L7 segments), in preparations in which the dorsal columns were transected between the L5 and L6 segments, whereas interneurons investigated in this study were located primarily in the L4 segment. Both subpopulations are coexcited by group I and II afferents, but group I input is dominant in the caudal lumbar segments, whereas group II afferents provide much stronger input to interneurons in the midlumbar segments (Harrison and Jankowska, 1985a,b; Edgley and Jankowska, 1987b; Riddell and Hadian, 2000). A more important contribution of midlumbar interneurons to the inhibition of DSCT neurons by group II afferents is also indicated by the muscle origin of this inhibition; it was most frequently evoked from the Q, Sart, FDL, and DP group II afferents, which are the main source of group II input to laminas V-VI midlumbar interneurons (Edgley and Jankowska, 1987b). More frequent IPSPs evoked in DSCT neurons from group I afferents in the GS and FDL nerves could accordingly be linked to the more potent input from group I afferents in these nerves to more caudally located interneurons (Harrison and Jankowska, 1985a).

Disynaptic inhibition of DSCT neurons via the same interneurons that mediate inhibition of hindlimb motoneurons from group II muscle afferents would strengthen the previous conclusions that not only ventral spinocerebellar tract neurons (Lundberg, 1971; Lindström, 1973) but also DSCT neurons (Hongo et al., 1983a,b) monitor inhibitory input to motoneurons provided by premotor interneurons in reflex pathways from peripheral afferents. Osborn and Poppele (1983) reported that inhibition of DSCT neurons is the most common response to muscle contractions and that many DSCT neurons respond in the same way to muscle stretch and to its contraction. It has also been proposed that one of the functions of DSCT neurons is to integrate information on muscle stretches and contractions with other kinds of information, before it is forwarded to the cerebellum and used to adjust motor activity by the cerebellum (Bosco and Poppele, 2001). The results of the present study are in full support of this conclusion but also show that the information forwarded by DSCT neurons is highly integrated already before it reaches DSCT neurons. As shown previously, inhibitory interneurons that modulate activity of DSCT neurons integrate information from tendon organs, muscle spindle primaries and secondaries, and a variety of other receptors as well as on descending commands (for references, see Jankowska, 1992; Davies and Edgley, 1994; Cabaj et al., 2006).

Finally, we want to draw attention to another consequence of results of this study: to the indications that differences between the populations of interneurons that mediate disynaptic actions from tendon organ (group Ib) afferents and of interneurons that mediate disynaptic actions from secondary muscle spindle (group II) afferents to motoneurons are becoming blurred and that these interneurons may be parts and parcel of the same interneuronal population rather than belong to two distinct populations. This would have serious consequences not only for the interpretation of information forwarded by these interneurons to the cerebellum but also for the understanding of their role in motor behavior and for any models of spinal neuronal networks contributing to various forms of movement, including postural adjustments as well as scratching or locomotion. A more detailed discussion of these consequences would nevertheless be outside the scope of this report.

\section{References}

Asif M, Edgley SA (1992) Projections of group II-activated midlumbar spinocerebellar tract neurones to the region of nucleus $\mathrm{Z}$ in the cat. J Physiol (Lond) 448:565-578.

Bannatyne BA, Edgley SA, Hammar I, Jankowska E, Maxwell DJ (2003) Networks of inhibitory and excitatory commissural interneurons mediating crossed reticulospinal actions. Eur J Neurosci 18:2273-2284. 
Bannatyne BA, Edgley SA, Hammar I, Jankowska E, Maxwell DJ (2006) Differential projections of excitatory and inhibitory dorsal horn interneurons relaying information from group II muscle afferents in the cat spinal cord. J Neurosci 26:2871-2880.

Bloedel JR, Courville J (1981) Cerebellar afferent systems. In: Handbook of physiology. The nervous system. Motor Control, pp 735-829. Bethesda, MD: American Physiological Society.

Bosco G, Poppele RE (2001) Proprioception from a spinocerebellar perspective. Physiol Rev 81:539-568.

Bras H, Cavallari P, Jankowska E, Kubin L (1989) Morphology of midlumbar interneurones relaying information from group II muscle afferents in the cat spinal cord. J Comp Neurol 290:1-15.

Brink E, Harrison PJ, Jankowska E, McCrea DA, Skoog B (1983) Postsynaptic potentials in a population of motoneurones following activity of single interneurones in the cat. J Physiol (Lond) 343:341-359.

Cabaj A, Stecina K, Jankowska E (2006) Same spinal interneurons mediate reflex actions of group Ib and II afferents and crossed reticulospinal actions. J Neurophysiol 95:3911-3922.

Cavallari P, Edgley SA, Jankowska E (1987) Post-synaptic actions of midlumbar interneurones on motoneurones of hind-limb muscles in the cat. J Physiol (Lond) 389:675-689.

Curtis DR, Eccles JC, Lundberg A (1958) Intracellular recording from cells in Clarke's column. Acta Physiol Scand 43:303-314.

Davies HE, Edgley SA (1994) Inputs to group II-activated midlumbar interneurones from descending motor pathways in the cat. J Physiol (Lond) 479:463-473.

Eccles JC, Eccles RM, Lundberg A (1957) Synaptic actions in motoneurones caused by impulses in Golgi tendon afferents. J Physiol (Lond) 138:227-252.

Eccles JC, Oscarsson O, Willis WD (1961) Synaptic action of group I and II afferent fibres of muscle on the cells of the dorsal spinocerebellar tract. J Physiol (Lond) 158:517-543.

Edgley SA, Jankowska E (1987a) Field potentials generated by group II muscle afferents in the middle lumbar segments of the cat spinal cord. J Physiol (Lond) 385:393-413.

Edgley SA, Jankowska E (1987b) An interneuronal relay for group I and II muscle afferents in the midlumbar segments of the cat spinal cord. J Physiol (Lond) 389:647-674.

Fuller JH, Schlag JD (1976) Determination of antidromic excitation by the collision test: problems of interpretation. Brain Res 112:283-298.

Gustafsson B, Jankowska E (1976) Direct and indirect activation of nerve cells by electrical pulses applied extracellularly. J Physiol (Lond) 258:33-61.

Harrison PJ, Jankowska E (1985a) Sources of input to interneurones mediating group I non-reciprocal inhibition of motoneurones in the cat. J Physiol (Lond) 361:379-401.

Harrison PJ, Jankowska E (1985b) Organization of input to the interneu- rones mediating group I non-reciprocal inhibition of motoneurones in the cat. J Physiol (Lond) 361:403-418.

Hongo T, Jankowska E, Ohno T, Sasaki S, Yamashita M, Yoshida K (1983a) Inhibition of dorsal spinocerebellar tract cells by interneurones in upper and lower lumbar segments in the cat. J Physiol (Lond) 342:145-159.

Hongo T, Jankowska E, Ohno T, Sasaki S, Yamashita M, Yoshida K (1983b) The same interneurones mediate inhibition of dorsal spinocerebellar tract cells and lumbar motoneurones in the cat. J Physiol (Lond) 342:161-180.

Jack JJB (1978) Some methods for selective activation of muscle afferent fibres. In: Studies in neurophysiology (Porter R, ed), pp 155-176. Cambridge, UK: Cambridge UP.

Jankowska E (1992) Interneuronal relay in spinal pathways from proprioceptors. Prog Neurobiol 38:335-378.

Jankowska E, Roberts WJ (1972) An electrophysiological demonstration of the axonal projections of single spinal interneurones in the cat. J Physiol (Lond) 222:597-622.

Jankowska E, Edgley SA, Krutki P, Hammar I (2005) Functional differentiation and organization of feline midlumbar commissural interneurones. J Physiol (Lond) 565:645-658.

Jansen JK, Nicolaysen K, Walloe L (1967) On the inhibition of transmission to the dorsal spinocerebellar tract by stretch of various ankle muscles of the cat. Acta Physiol Scand 70:362-368.

Krutki P, Jankowska E, Edgley SA (2003) Are crossed actions of reticulospinal and vestibulospinal neurons on feline motoneurons mediated by the same or separate commissural neurons? J Neurosci 23:8041-8050.

Lindström S (1973) Recurrent control from motor axon collaterals of Ia inhibitory pathways in the spinal cord of the cat. Acta Physiologica Scandinavica 89 [Suppl 392]:1-43.

Lindström S, Takata M (1977) Lack of recurrent depression from motor axon collaterals of Ia IPSPs in dorsal spinocerebeller tract neurones. Brain Res 129:158-161.

Lundberg A (1971) Function of the ventral spinocerebellar tract. A new hypothesis. Exp Brain Res 12:317-330.

Osborn CE, Poppele RE (1983) Cross-correlation analysis of the response of units in the dorsal spinocerebellar tract (DSCT) to muscle stretch and contraction. Brain Res 280:339-342.

Riddell JS, Hadian M (2000) Interneurones in pathways from group II muscle afferents in the lower- lumbar segments of the feline spinal cord. J Physiol (Lond) 522:109-123.

Riddell JS, Jankowska E, Eide E (1993) Depolarization of group II muscle afferents by stimuli applied in the locus coeruleus and raphe nuclei of the cat. J Physiol (Lond) 461:723-741.

Riddell JS, Jankowska E, Huber J (1995) Organization of neuronal systems mediating presynaptic inhibition of group II muscle afferents in the cat J Physiol (Lond) 483:443-460.

Roberts WJ, Smith DO (1973) Analysis of threshold currents during microstimulation of fibres in the spinal cord. Acta Physiol Scand 89:384-394. 